\title{
Pregnant Women's Knowledge Gaps about Breastfeeding in Northern Portugal
}

\author{
Alexandrina Cardoso ${ }^{1 *}$, Abel Paiva e Silva1, Heimar Marín ${ }^{2}$ \\ ${ }^{1}$ Escola Superior de Enfermagem do Porto (Porto School of Nursing), Porto, Portugal \\ ${ }^{2}$ Federal de São Paulo University, São Paulo, Brasil \\ Email: ^alex@esenf.pt, abel@esenf.pt, heimar@denf.epm.br
}

How to cite this paper: Cardoso, A., e Silva, A.P. and Marín, H. (2017) Pregnant Women's Knowledge Gaps about Breastfeeding in Northern Portugal. Open Journal of Obstetrics and Gynecology, 7, 376- 385. https://doi.org/10.4236/ojog.2017.73039

Received: February 22, 2017

Accepted: March 26, 2017

Published: March 29, 2017

Copyright (c) 2017 by authors and Scientific Research Publishing Inc. This work is licensed under the Creative Commons Attribution International License (CC BY 4.0).

http://creativecommons.org/licenses/by/4.0/

Open Access

\begin{abstract}
Introduction: To explore Portuguese pregnant women' knowledge on breastfeeding and to correlate knowledge scores with the socio-demographic variables. Methods: A cross-sectional design was used to assess 621 pregnant women' knowledge on breastfeeding at three health centers in the North of Portugal. The face-to-face interviewing was used to collected data using a clinical instrument composed by 18 knowledge breastfeeding descriptors. The reliability of the instrument was very good $(K R-20=0.88)$. Descriptive and inferential statistics were used to examine pregnant women' knowledge and the relation with socio-demographics variables. Results: Pregnant women were found to be more knowledgeable in benefits of breastfeeding. In 14 knowledge descriptors more than $60 \%$ of pregnant women revealed lack of knowledge. The most usual lack of knowledge concerned how to increase lactation and how to deal with breastfeeding complications. The pregnant women more knowledgeable were the oldest, with higher education, and with previous experience on breastfeeding. Discussion: Pregnant women revealed a significant lack of knowledge to support basic breastfeeding decisions. The focus of care should emphasize on the younger, the first-time mothers and less well-educated women as particular vulnerable and poorer prepared groups concerning breastfeeding knowledge.
\end{abstract}

\section{Keywords}

Breastfeeding, Nursing-Midwife Assessment, Pregnant Women, Midwifery

\section{Introduction}

Breastfeeding is a healthier choice for the baby, mother and family [1] [2]. However, breastfeeding rates for exclusive breastfeeding have not reached the goals established by health organizations in several countries. 
Breastfeeding can be emotionally demanding and physically exhausting. For many women, breastfeeding is not instinctive, but rather a learned process. Therefore, mothers need to begin the preparation for breastfeeding during pregnancy. To breastfeed with success mothers needs to learn about proper position, latch, sucking and signs of milk transfer, hunger cues, and the infant's receptiveness to breastfeeding [1] [3].

Almost all mothers experience doubts about their ability to care for their newborns, particularly if they are breastfeeding. The lack of knowledge and confidence may jeopardize the success to breastfeeding. Studies have highlighted that a mother's knowledge and skills can increase the rate and duration of breastfeeding and are a relevant component of effective decisions and actions related to breastfeeding. [4]-[9] Several studies suggest that the main factors associated with short duration of breastfeeding were maternal ones. Specifically concerning knowledge on breastfeeding, the study reported that poor maternal knowledge was a relevant risk factor for breastfeeding abandonment. [10] [11] [12] [13]

Chezem et al. [14] explored relationships among breastfeeding knowledge, breastfeeding confidence, and infant feeding plans and their effects on feeding practices in first-time breastfeeding mothers. The authors concluded that breastfeeding knowledge was strongly correlated with breastfeeding confidence and lactation duration.

Lack of knowledge on breastfeeding could lead to hasty decisions and may also hamper the way mothers handle situations that may arise. Several studies reported that perceived inadequate milk supply was the most common reason for introducing formula feeding, and the most common problems encountered were fatigue, sore nipples, and engorgement. [12] [13] They suggested that breast engorgement and fatigue could be effectively managed with increased knowledge about positioning, alternating breasts with each feeding and resting when the baby rests. The participants inadequate lactation knowledge was demonstrated when they failed to mention the recognized indicators of breastfeeding problems, such as diaper count, listlessness of the baby, or failure to let down, as reasons for discontinuing breastfeeding. They still demonstrated the importance of correcting the sucking techniques to prevent problems, such as sore or cracked nipples. The authors postulated that women needed to be better prepared on breastfeeding to prevent and manage problems that could lead to discontinuation of breastfeeding.

Carlisle [15] described the relationship between the knowledge on breastfeeding issues and the success of a mother's ability to breastfeed for three months or longer. The author found that mothers were more likely to overcome challenges, such as cluster feeding or alterations in sleep pattern, if they were informed and prepared for these changes.

Nurses and midwives are important in the promotion, protection and support of breastfeeding. [2] [16] According to the literature, antenatal education is an important factor in developing breastfeeding knowledge development and fos- 
tering breastfeeding skills and confidence for initiating and maintaining breastfeeding until the sixth month, as currently recommended. [2] [4]

Nevertheless, Chaudhary et al. [9] argued that maternal knowledge about breastfeeding was inadequate, and there was a big gap between actual and desired practices. In turn, others authors claimed that increasing mothers' knowledge was the first step to increase breastfeeding rates and duration.

To foster the mothers' preparation for breastfeeding, a systematized assessment of pregnant women' learning needs/knowledge concerning breastfeeding is needed. What do pregnant women know about breastfeeding during pregnancy? What is the level of pregnant knowledge about breastfeeding? Are there differences in pregnant women' levels of knowledge in relation to age, education, parity, planned pregnancy and previous experience on breastfeeding? So, to understand Portuguese pregnant women' knowledge on breastfeeding, was developed a study that aims to characterize expectant mothers' knowledge and examine scores of breastfeeding knowledge in relation to parental demographics.

\section{Methods}

\subsection{Design}

A cross-sectional study was developed in three Health Centres in North of Portugal, with previous authorization of Ethics Committee of the institutions.

\subsection{Participants and Sample}

Participants were recruited during their visit to the Health Centre for pregnancy appointment. The convenience sample was constituted for pregnant women whose foetus was 24 - 28 gestational age, over 18 years of age, and fluent in Portuguese, resulting in a sample size of 621 pregnant women who consented to the study.

\subsection{Procedures}

The data were collected by the researcher that who a nurse-midwife from January to July of 2011, using face-to-face interview. Data collection used an instrument designed for this purpose, but based on nursing descriptors described in Nursing Outcomes Classification (NOC). [17] The instrument that guide the assessment of pregnant women' breastfeeding knowledge included 18 knowledge descriptors (Figure 1).

The instrument was composed of two parts: a) socio demographic and obstetric data and sources of information; b) knowledge instrument, with the 18 knowledge descriptors. Each knowledge descriptor was documented according to researcher clinical judgment on the pregnant women' knowledge on the specific topic. Thus, if pregnant women revealed the knowledge, their response was recorded as "yes" meaning adequate knowledge, or if they did not reveal the knowledge, the response was recorded as "no" meaning lack of knowledge of breastfeeding. [18] For instance, to assess pregnant' knowledge about proper latch signs, they were asked "How do you think you know that the baby is doing 


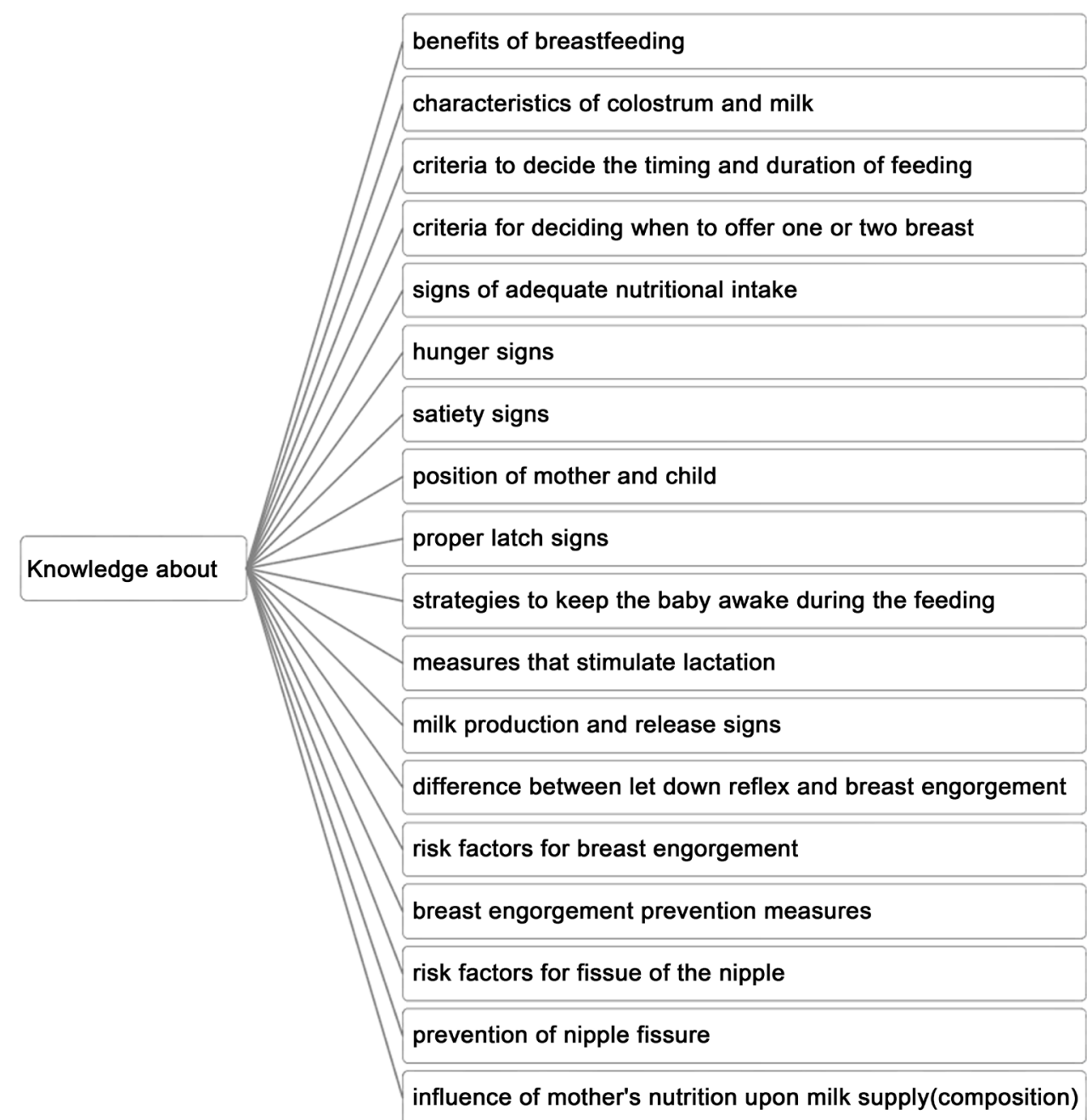

Figure 1. Descriptors used to assess pregnant women' breastfeeding knowledge.

a good latch?" If the pregnant women mentioned that "the baby's chin should touch the breast, but the nose should not, and the baby lips should cover more of the areola with the lower lip than with the upper lip" their response was recorded as "yes", meaning they had adequate knowledge about proper latch signs.

The instrument reliability was analyzed by calculating the Kuder-Richardson Coefficient (KR-20), indicated to test the reliability of binary measurements. There was very good internal reliability $(\mathrm{K}-\mathrm{R} 20=0.88)$.

\subsection{Data Analysis}

To analyze the data, the Statistical Package for Social Sciences (SPSS) program version 19.0 was used. The pregnant women' knowledge was analyzed using descriptive statistics, and the profile of expectant mothers more knowledgeable about breastfeeding was analyzed using inferential statistics. The score of maternal knowledge on breastfeeding was calculated, ranging between 0 and 1 .

The scores of breastfeeding knowledge and their relationships with maternal characteristics (i.e., age, education level, living with the child's father, parity, planned pregnancy and previous experience on breastfeeding) were examined. 


\subsection{Ethical Considerations}

The ethical principles in the Declaration of Helsinki were used in the planning and conduct of the study. The institutions involved gave the approval and nurses-midwives of health centers were approached to facilitate recruitment of pregnant women. Eligible pregnant women provided voluntary consent for participation after researcher explanation of the study purpose, procedures, benefits and that declining participation would have no adverse consequences in health care provision. All participants were assured of confidentiality. After each interview, the researcher shared with nurse the main nursing diagnosis to contribute to continuity of care.

\section{Results}

The mean age of the 621 expectant mothers that participated in the study was $29.55(\mathrm{SD}=5.23)$. In relation to education level, $42.0 \%$ of pregnant women had higher education level, 32.7\% had between 9 and 12 years of education and $25.3 \%$ had less than 9 years of education. For $62.9 \%$ of women the pregnancy was planned. More than a quarter of pregnant women already had one child (28.2\%). Almost all the pregnant women revealed intention to breastfeeding (99.3\%) and $25.1 \%$ referred to previous breastfeeding experience.

Regarding sources of information, the results highlighted that pregnant women used more formal than informal sources. In fact, more than half of the pregnant women' revealed that utilized as sources of information related to breastfeeding: nurse-midwife (51.1\%); medical doctor (50.6\%); books (55.0\%); and internet $(50.8 \%)$. Family and others mothers were referred as source of information for $27.6 \%$ and $19.9 \%$, respectively.

The examination of pregnant women 'knowledge about breastfeeding revealed that they were knowledgeable about the benefits of breastfeeding (69.9\%). In the assessment of the others 17 descriptors, more than half of the pregnant women' revealed a lack of knowledge (Table 1 ).

For five knowledge descriptors, more than $80 \%$ of the pregnant women revealed lack of knowledge. In fact, $86.5 \%$ of the pregnant women revealed a lack of knowledge about the measures that stimulate lactation, $80.5 \%$ revealed a lack of knowledge about the signs of milk production and release and $78.3 \%$ revealed a lack of knowledge about signs of adequate nutritional intake.

Fissures of the nipple and breast engorgement were complications often associated with breastfeeding in the early weeks, and the main causes of breastfeeding failure and the most frequent causes of breastfeeding discontinuation. As observed in Table 1, more than three-quarters of the pregnant women revealed a lack of knowledge concerning breast engorgement and nipple fissure risk factors and prevention measures.

Pregnant women who had a higher knowledge level were the oldest $(\mathrm{U}=$ 37,591.50; $P=0.007)$, already had another child $(\mathrm{U}=22,246.50 ; P<0.001)$ and cohabited with the father of the child $(U=16,257.50 ; P<0.001)$. There was also a significant difference in the level of pregnant women' breastfeeding knowledge 
Table 1. Percentage of pregnant women with lack of knowledge on breastfeeding descriptors.

\begin{tabular}{lc}
\hline \multicolumn{1}{c}{ Knowledge of... } & Lack of knowledge \% (N) \\
\hline Benefits of breastfeeding & $28.7(178)$ \\
Satiety signs & $55.2(343)$ \\
Hunger signs & $55.6(345)$ \\
Position of mother and child & $56.2(349)$ \\
Strategies to keep the baby awake during the feeding & $62.5(388)$ \\
Characteristics of colostrum and milk & $63.3(393)$ \\
Influence of mother's nutrition upon milk supply (composition) & $67.2(416)$ \\
Criteria to decide the timing and duration of breastfeeding & $68.8(428)$ \\
Proper latch signs & $73.4(456)$ \\
Criteria for deciding when to offer one or two breast & $74.2(461)$ \\
Risk factors for fissure of the nipple & $77.5(481)$ \\
Prevention of nipple fissure & $77.5(481)$ \\
Signs of adequate nutritional intake & $78.3(486)$ \\
Milk production and release signs & $80.5(500)$ \\
Breast engorgement prevention measures & $80.5(500)$ \\
Risk factors for breast engorgement & $81.5(506)$ \\
Difference between let down reflex and breast engorgement & $83.6(519)$ \\
Measures that stimulate lactation & $86.5(537)$ \\
\hline
\end{tabular}

by education degree $\left(\chi_{(2)}^{2}=16.24 ; P<0.001\right)$, confirming that pregnant women with higher education levels had higher knowledge levels than pregnant women with basic or secondary education.

There was a significant difference in pregnant women' knowledge level $(\mathrm{U}=$ 201,20.50; $P<0.001)$ concerning previous experience on breastfeeding, confirming that mothers who reported having previous experience demonstrated a higher knowledge level.

\section{Discussion}

The lack of knowledge can influence the success and duration of breastfeeding. There is consensus in the literature that the knowledge and skills to ensure the correct latch and to adopt measures to stimulate the lactation are essential to effective breastfeeding. [1] [2] [3] The importance assigned by pregnant women to learn about breastfeeding is reported in several studies. The results in this study also found that pregnant women had a significant lack of knowledge.

\subsection{Lack of Knowledge Related to Lactation Stimulation}

In actual study was observed lack of knowledge particularly concerning lactation stimulation. A total of $86.7 \%$ expectant mothers revealed lack of knowledge of measures that stimulate lactation and $80.5 \%$ revealed lack of knowledge of milk 
production and release signs. This two indicators were the ones that more pregnant revealed deficit. In a Finnish study was observed similar results. Laanterä et al. [12] in their study that aimed to describe breastfeeding knowledge of childbearing parents including 123 pregnant mothers and 49 fathers verified that the respondents correctly answered $68 \%$ of the items related to breastfeeding knowledge.

In the Percegoni et al. [19] study, 62.2\% of mothers recognized the existence of factors that interfered with the production and release of breast milk, but only $1.5 \%$ referred to the importance of proper latch as one of these factors. Chaudhary et al. [9] observed that 75\% revealed a lack of knowledge about the meaning of exclusive breastfeeding and the importance of night feeding. Ekambaram et al. [8] reported that the maternal knowledge was inadequate regarding time to initiate breastfeeding (92\%), colostrum feeding (56\%), duration of exclusive breastfeeding (38\%), and expressed breast milk (51\%).

Contrariwise, Senarath et al. [5] highlighted different results. The authors found that more than $90 \%$ of mothers knew that breastfeeding should be offered on demand, the advantages of colostrum, and the recommended duration for exclusive breastfeeding.

\subsection{Lack of Knowledge Related to Breastfeeding Technique}

Three-quarters of pregnant women didn't know the signs of proper latch and the criteria for how to decide to offer one or both breasts at each feeding, and $86.5 \%$ did not demonstrate knowledge about lactation stimulation measures. Those findings are analogous to the results of other studies. In fact, Percegoni et al. [19] found that $61 \%$ and $79 \%$ of mothers, respectively, did not demonstrate knowledge about how to ensure the proper latch.

\subsection{Lack of Knowledge Related to Complications}

$\begin{array}{lr}\text { Risk factors for fissure of the nipple } & 77.5(481) \\ \text { Prevention of nipple fissure } & 77.5(481) \\ \text { Signs of adequate nutritional intake } & 78.3(486) \\ \text { Breast engorgement prevention measures } & 80.5(500) \\ \text { Risk factors for breast engorgement } & 81.5(506) \\ \text { Difference between let down reflex and breast engorgement } & 83.6(519)\end{array}$

The lack of knowledge, particularly about breastfeeding technique, about the intervals of feeding, and about the use pacifier and/or silicone nipples, with the consequent inability to ensure a proper latch, constitute risk factors for breastfeeding complications and consequent abandonment of breastfeeding. The evidence shows that a higher knowledge level about breastfeeding and complications management has the potential to lead to an increase in the duration of breastfeeding and to produce more positive perceptions about breastfeeding experience. 


\subsection{Profileof Knowledgeable Pregnant}

In this study, the pregnant women who had demonstrated a higher knowledge level were the oldest, had higher education level, and had previous experience in breastfeeding. These findings were similar to the results of other studies. [5] [6] [8] [12] [19]

Berman [20] reported that pregnant women ranked breastfeeding fourth among content considered most important to learn during pregnancy. In the study, $60 \%$ of first-time mothers considered breastfeeding "very important"; however, among mothers who already had one child, only $28.2 \%$ considered "very important". In this study, mothers with previous experience on breastfeeding were those who revealed higher knowledge level.

In this study, pregnant women revealed a reasonable level of breastfeeding knowledge. This result is similar to results reported in other studies. Chaudhary et al. [9] reported that mothers did not have adequate knowledge about the correct way to breastfeed. In other studies, the knowledge level was satisfactory (Senarath et al.) or fairly good (Radzniwan et al.) [5] [21] Also, Kishore et al. (2009) stated that $39 \%$ of mothers had satisfactory breastfeeding knowledge. [22] Komarsson et al. [6] found that mothers demonstrated a good knowledge level about breastfeeding issues.

The majority of pregnant women in this study chose to breastfeed their babies, perhaps because of their knowledge about breastfeeding benefits for the child and mother. In fact, only $28.7 \%$ of the pregnant women revealed a lack of knowledge on this topic. Other authors have reported similar results. According to Zhou et al. (2010), the knowledge that "breastfeeding is better for the baby" was indicated as the main reason for breastfeeding (87\%). [11] However, Radzniwan et al. [21] reported that more than $20 \%$ of mothers in their study were ignorant regarding breast milk protection against allergies, and $15 \%$ of mothers were not aware that breast milk is better than formula. Chaudhary et al. [9] reported in their study that only $25 \%$ had an idea about the importance of colostrum.

Based on the low breastfeeding knowledge level, the results of this study confirmed that mothers need to learn how to breastfeed their babies, and that the learning process should be initiated during pregnancy. The evidence has shown that antenatal education on breastfeeding has a positive effect on the duration of breastfeeding. Other studies corroborate those results, verifying that antenatal education has a positive impact on breastfeeding initiation and maintenance rates. [4] [8]

\section{Conclusions}

Pregnant women revealed a significant lack of knowledge at the moment when it was assessed.

The expectant mothers more knowledgeable are the oldest, with higher education levels, cohabited with the father of the child, and those that already had another child and breastfeeding experience. The focus of care should emphasize 
on the younger, the first-time mothers and less well-educated women as particular vulnerable and poorer prepared groups concerning breastfeeding knowledge. The challenge is to prepare mothers to breastfeeding decisions and actions, beginning in pregnancy. Increasing pregnant women' knowledge and confidence in their capacity to decide and act will contribute to mastery and breastfeeding success.

\section{References}

[1] World Health Organization (WHO) (2009) Child Health and Development Evidence for Ten Steps to Successful Breastfeeding. Family and Reproductive Health Division of Child Health and Development, Geneva.

[2] Association of Women's Health, Obstetric and Neonatal Nurses (2007) Breastfeeding Support: Prenatal Care through the First Year. In: AWHONN, Ed., Evidence-Based Clinical Practice Guideline. AWHONN, Washington, 89p.

[3] Mulder, P.J. (2006) A Concept Analysis of Effective Breastfeeding. Journal of Obstetric, Gynecologic, \& Neonatal Nursing, 35, 332-339.

https://doi.org/10.1111/j.1552-6909.2006.00050.x

[4] Dyson, L., McCormick, F. and Renfrew, M.J. (2005) Interventions for Promoting the Initiation of Breastfeeding. Cochrane Database Systematic Reviews, 2, 1-23. https://doi.org/10.1002/14651858.cd001688.pub2

[5] Senarath, U., Fernando, D., Vimpani, G. and Rodrigo, I. (2007) Factors associated with maternal knowledge of newborn care among hospital-delivered mothers in Sri Lanka. Transactions of the Royal Society of Tropical Medicine and Hygiene, 101, 823-830. https://doi.org/10.1016/j.trstmh.2007.03.003

[6] Komarsson, K., Oriá, M., Dodt, R., Almeida, P. and Ximenes, L. (2008) Mother's knowledge about breastfeeding: a descriptive study. Online Brazilian Journal of Nursing, 7.

http://www.objnursing.uff.br/index.php/nursing/article/view/j.1676-4285.2008.1558.

[7] Wen LM, Baur LA, Rissel C, Alperstein G, and Simpson JM. (2009) Intention to Breastfeed and Awareness of Health Recommendations: Findings from First-Time Mothers in Southwest Sydney, Australia. International Breastfeeding Journal, 4, 9. https://doi.org/10.1186/1746-4358-4-9

[8] Ekambaram, M., Bhat, V.B. and Ahamed, M.A. (2010) Knowledge, Attitude and Practice of Breastfeeding among Postnatal Mothers. Current Pediatric Research, 14, 119-124.

[9] Chaudhary, R.N., Shah, T. and Raja, S. (2011) Knowledge and Practice of Mothers Regarding Breast Feeding: A Hospital Based Study. Health Renaissance, 9, 194-200. https://doi.org/10.3126/hren.v9i3.5590

[10] Chambers, J.A., Mclnnes, R.J., Hoddinott, P., and Alder, E.M. (2007) A Systematic Review of Measures Assessing Mothers' Knowledge, Attitudes, Confidence and Satisfaction towards Breastfeeding. Breastfeed Review, 15, 17-25.

[11] Zhou, Q., Younger, K., John, M. and Kearney, M. (2010) An Exploration of the Knowledge and Attitudes towards Breastfeeding among a Sample of Chinese Mothers in Ireland. BMC Public Health, 10, 722. https://doi.org/10.1186/1471-2458-10-722

[12] Laanterä, S., Pietilä, A. and Pölkki, T. (2010) Knowledge of Breastfeeding among Pregnant Mothers and Fathers. Journal of Perinatal \& Neonatal Nursing, 24, 320329. https://doi.org/10.1097/JPN.0b013e3181ec0c60 
[13] Brand, E., Kothari, C. and Stark, M.A. (2011) Factors Related to Breastfeeding Discontinuation between Hospital Discharge and 2 Weeks Postpartum. The Journal of Perinatal Education, 20, 36-44. https://doi.org/10.1891/1058-1243.20.1.36

[14] Chezem, J., Friesen, C. and Boettcher, J. (2003) Breastfeeding Knowledge, Breastfeeding Confidence, and Infant Feeding Plans: Effects on Actual Feeding Practices. Journal of Obstetric, Gynecologic, \& Neonatal Nursing, 32, 40-47. https://doi.org/10.1177/0884217502239799

[15] Carlisle, D. (2008) Breastfeeding Friendly. Community Practice, 81, 10-11.

[16] Cardoso, A. and Paiva e Silva, A. (2010) Representing Nursing Knowledge on Maternal and Neonatal Health. A Study on the Cultural Suitability of ICNP. International Nursing Review, 57, 426-434. https://doi.org/10.1111/j.1466-7657.2010.00829.x

[17] Moorhead, S.A., Johnson, M. and Maas, M., Eds. (2008) Nursing Outcomes Classification. 4rd Edition, Mosby, St. Louis, MO.

[18] International Council of Nurses (2012) International Classification for Nursing Practice 2011. Geneva. http://www.icn.ch/ICNP-Browser-NEW.html

[19] Percegoni, N., Araújo, R., Silva, M., Euclydes, M.P., Tinôco, A. (2002) Knowledge on Breastfeeding Mothers Attended at Two Hospitals in Viçosa, Minas Gerais (Article in Portuguese). Revista de Nutrição, 15, 29-35. https://doi.org/10.1590/S1415-52732002000100004

[20] Berman, R.O. (2006) Perceived Learning Needs of Minority Expectant Women and Barriers to Prenatal Education. The Journal of Perinatal Education, 15, 36-42. https://doi.org/10.1624/105812406X107807

[21] Radzniwan, A., Azimah, N., Zuhra, H., Khairani, O. (2009) Breast Feeding Practice and Knowledge among Mothers Attending an Urban Malaysian Maternal and Child Health Clinic. Med Health, 4, 1-7.

[22] Kishore, M.S., Kumar, P., Aggarwal, A.K. (2009) Breastfeeding Knowledge and Practices amongst Mothers in a Rural Population of North India: A Community-Based Study. Journal of Tropical Pediatrics, 55, 183-188. https://doi.org/10.1093/tropej/fmn110

Submit or recommend next manuscript to SCIRP and we will provide best service for you:

Accepting pre-submission inquiries through Email, Facebook, LinkedIn, Twitter, etc. A wide selection of journals (inclusive of 9 subjects, more than 200 journals)

Providing 24-hour high-quality service

User-friendly online submission system

Fair and swift peer-review system

Efficient typesetting and proofreading procedure

Display of the result of downloads and visits, as well as the number of cited articles

Maximum dissemination of your research work

Submit your manuscript at: http://papersubmission.scirp.org/

Or contact ojog@scirp.org 